\title{
Loop Cosmology: Regularization vs. Quantization
}

\author{
Jaume Haro $^{1, *}$ and Emilio Elizalde ${ }^{2, \dagger}$ \\ ${ }^{1}$ Departament de Matemàtica Aplicada I, Universitat Politècnica \\ de Catalunya, Diagonal 647, 08028 Barcelona, Spain
}

${ }^{2}$ Instituto de Ciencias del Espacio (CSIC) and Institut d'Estudis Espacials de Catalunya (IEEC/CSIC), Facultat de Ciències, UAB, Torre C5-Parell-2a planta, 08193 Bellaterra (Barcelona) Spain

It is argued that it is the regularization of the classical Hamiltonian-first step in loop cosmology in order to build a well-defined quantum theory—what is already able to avoid the Big Bang and Big Rip singularities, rather than the usually invoked quantum corrections coming from the quantization of the Hamiltonian. To prove such statement, the classical regularized Hamiltonian corresponding to loop gravity is obtained, and it is shown that it coincides, up to terms of order $\hbar$, with the so-called effective Hamiltonian which is calculated from the quantum regularized Hamiltonian using semi-classical techniques. From that comparison it is concluded that both types of singularities are avoided at the "classical level" already, i.e., using loop cosmology, in the sense that, only the quantum nature of the geometry is invoked (the loop cut off) in order to construct the regularized Hamiltonian and to fix the parameter on which it depends. Such finding constitutes a key manifestation of the intrinsic power of loop gravity, as compared with other alternatives.

PACS numbers: 98.80.Jk, 98.80.Qc, 04.60.Pp, 04.60.Ds

\section{INTRODUCTION}

In this paper we review some features used in loop quantum cosmology (LQC) to get rid of the classical Big Bang and Big Rip singularities in Friedmann-Robertson-Walker (FWR) cosmologies. We will show in what follows that, using the classical regularized Hamiltonian introduced in loop gravity in order to construct a well-defined quantum theory [1-4], these singularities are already

\footnotetext{
* E-mail: jaime.haro@upc.edu

$\dagger$ E-mail: elizalde@ieec.fcr.es, elizalde@math.mit.edu
} 
avoided, without the need to quantify this Hamiltonian. To prove such statement, that may come as a surprise, we first review the so-called old quantization of loop quantum cosmology $[5,6]$ based on holonomies of the form $h_{j}(\mu) \equiv e^{-i \frac{\mu x}{2 c} \sigma_{j}}$ [7], where $\sigma_{j}$ are the Pauli matrices, $c$ denotes the velocity of light, $\mu$ is a dimensionless parameter, and $x \equiv \iota \dot{a}$, being $\iota$ the Barbero-Immirzi parameter and $\dot{a}$ the scale factor velocity. To fix the value of the parameter $\mu$ one can use the quantum nature of the geometry (the minimum eigenvalue of the area operator introduced in loop quantum geometry), and it is at this very point when the quantum nature of the geometry explicitly appears. In this framework we obtain, at the classical level (i.e. using the regularized Hamiltonian without quantizing it in any way), the modified Friedmann equation that has no Big Bang singularity for barotropic fluids with an equation of state $p=\omega \rho$ satisfying $\omega>-1 / 3$.

To avoid the Big Bang singularity, for all values of $\omega$ for which this singularity appears in the standard cosmology (that is, for $\omega>-1$ ) one has to introduce new phase-space variables, namely $V \equiv a^{3}$ and $\beta \equiv \iota \dot{a} / a[8,9]$, and the holonomies $h_{j}(\mu) \equiv e^{-i \frac{\lambda \beta}{2 c} \sigma_{j}}$, where $\lambda$ is here a parameter with the dimensions of length, which we fix in the same way as the parameter $\mu$, that is, using the quantum nature of the geometry. With these variables, and using the regularized Hamiltonian just at the classical level, one obtains a new modified Friedmann equation where it can be shown explicitly that the Big Bang singularity is completely avoided. Moreover, using this modified Friedmann equation, it is also possible to prove that, when $\omega<-1$, the corresponding Big Rip singularity can be avoided too [10-12].

We must stress the fact that both types of singularities are explicitly resolved just resorting to the classical regularized Hamiltonian. It is then rather clear that in the quantum version of the theory, that is, using the quantum Hamiltonian in LQC, the same features will emerge as in the classical one (the same results of the classical theory will be obtained, only with small modifications, of order $\hbar$ ) and, clearly, the singularities will also be absent here. In particular, using semi-classical techniques, coherent states, etc. [9, 13-15], an effective Hamiltonian is obtained from the quantum regularized Hamiltonian which explicitly coincides — up to modifications of order $\hbar$-with the classical regularized one. This is perhaps the reason why it was plainly concluded before that quantum effects, provided by LQC - that is, the effects due to the quantization of the regularized Hamiltonian-were the ones actually responsible for suppressing the singularity (see for example $[4,7,16]$ etc.). It is thus important to stress the following consequence of our study: the reason the singularities do not appear in LQC is because they already do not show up in the classical, regularized theory, i.e., in loop 
cosmology.

\section{OLD QUANTIZATION OF LOOP QUANTUM COSMOLOGY}

In the old quantization of LQC one considers the canonically conjugate variables $p \equiv a^{2}$ and $x \equiv \iota \dot{a}$ where $\iota$ is the Barbero-Immirzi parameter. Their Poisson bracket is $\{x, p\}=\frac{8 \pi G \iota}{3 c^{2}}=\frac{2 \iota}{\gamma^{2}}$, where the parameter $\gamma^{2} \equiv \frac{3 c^{2}}{4 \pi G}=\frac{3 \hbar}{4 \pi c l_{p}^{2}}$ has been introduced (being $G$ Newton's constant and $l_{p}$ the Planck length). One also considers the holonomies $h_{j}(\mu) \equiv e^{-i \frac{\mu x}{2 c} \sigma_{j}}=\cos \left(\frac{\mu x}{2 c}\right)-i \sigma_{j} \sin \left(\frac{\mu x}{2 c}\right)$ [7], where $\sigma_{j}$ are the Pauli matrices, $c$ denotes the velocity of light, and $\mu$ is a dimensionless parameter.

The key step in our procedure is the following. To get the gravitational part of the Hamiltonian, in the flat case, one cannot directly use the rather obvious one: $H_{\text {grav }}=-\frac{3 c^{2}}{8 \pi G \iota^{2}} x^{2} \sqrt{p}$, which actually leads to singular classical dynamics, because in LQC the operator $\hat{x}^{2}$ is not well-defined in the corresponding Hilbert space which is characterized by the fact that a generic element on it can be expanded as a countable sum of almost periodic functions of $x$ of the form $e^{i \beta x / 2}$, where $\beta$ is a real number. Then, since $x^{2}$ has not a well-defined quantum operator (in the correspondence principle), in order to construct an admissible quantum Hamiltonian operator one needs an almost periodic function of $x$ which approaches $x^{2}$ for small values of $x$. This can be actually constructed by using the general formulae of loop gravity that express the Hamiltonian in terms of holonomies and the variable $p$ [2-4]:

$$
\begin{gathered}
H_{\text {grav }, \text { reg }} \equiv-\frac{\hbar^{2} c}{32 \pi^{2} l_{p}^{4} \iota^{3} \mu^{3}} \sum_{i, j, k} \varepsilon^{i j k} \operatorname{Tr}\left[h_{i}(\mu) h_{j}(\mu) h_{i}^{-1}(\mu)\right. \\
\left.\times h_{j}^{-1}(\mu) h_{k}(\mu)\left\{h_{k}^{-1}(\mu), p^{3 / 2}\right\}\right]=-\frac{\gamma^{2} c^{2}}{2 \iota^{2}} a \sin ^{2} \frac{\mu x}{c}
\end{gathered}
$$

Note that this regularized Hamiltonian coincides, up to order $\hbar$, with the so-called effective Hamiltonian obtained from the quantum regularized Hamiltonian after use of the WKB approximation, path integral, or coherent state techniques [16]. But what is here to be remarked is that, in all papers devoted to the study of LQC, the authors start with the quantum version of the regularized Hamiltonian (a discrete theory) and then, in order to obtain a continuous one, they calculate via WKB approximation, or the like, the effective Hamiltonian which is, up to order $\hbar$, equal to $-\frac{\gamma^{2} c^{2}}{2 \iota^{2}} a \sin ^{2} \frac{\mu x}{c}$. We believe that this hard work could be avoided, and that can even produce some confusion, because it seems to indicate that the only way to describe the features of LQC within a continuous theory is to consider the effective Hamiltonian obtained from the quantum version of the theory (see for example [7, 16]). 
Taking now this regularized Hamiltonian as the gravitational part of the full one, the complete Hamiltonian is given by

$$
H_{r e g} \equiv-\frac{\gamma^{2} c^{2}}{2 \iota^{2} \mu^{2}} a \sin ^{2} \frac{\mu x}{c}+a^{3} \rho(a),
$$

where $\rho(a)$ is the matter density. The scalar factor satisfies the dynamic equation

$$
\dot{a}=\left\{a, H_{r e g}\right\}=\frac{c}{2 \iota \mu} \sin \frac{2 \mu x}{c},
$$

and imposing now the Hamiltonian constraint, $H_{r e g}=0$, we obtain the following modified Friedmann equation

$$
\frac{\dot{a}^{2}}{a^{2}}=\frac{2 \rho}{\gamma^{2}}\left(1-\frac{\rho(a)}{\rho_{c}(a)}\right)
$$

with the critical density being

$$
\rho_{c}(a) \equiv \frac{\gamma^{2} c^{2}}{2 \iota^{2} \mu^{2} a^{2}}=\frac{3 \hbar c}{8 \pi \iota^{2} \mu^{2} a^{2} l_{p}^{2}}=\frac{3 c^{4}}{8 \pi \iota^{2} \mu^{2} G a^{2}} .
$$

Here a remark is in order. To obtain a value for the parameter $\mu$, one quantizes the variables $x$ and $p$ as: $\hat{x}=x$ and $\hat{p}=-i \hbar \frac{2 \iota}{\gamma^{2}} \partial_{x}$. Since an eigenfunction of the operator $\hat{p}$ is $e^{-\frac{i \mu x}{2 c}}$, with eigenvalue $\frac{\hbar \iota \mu}{c \gamma^{2}}$, then, by identifying this eigenvalue with the minimum eigenvalue of the area operator in loop quantum gravity (LQG), which is $2 \sqrt{3} \pi \iota l_{p}^{2}$, one obtains $\mu=\frac{3 \sqrt{3}}{2}$ [4]. Note that we have actually fixed the value of $\mu$ just by invoking the quantum nature of the geometry, but what we have not done yet is to quantify the regularized Hamiltonian.

An important case, already discussed in [4], is when one considers a massless scalar field $\phi$. In this case the density is given by $\rho(a)=\frac{p_{\phi}}{2 a^{6}}$, where the constant $p_{\phi}$ is the momentum of the field. It is well-known that using the Friedmann equation one obtains a Big Bang singularity, however, using the modified one it is easy to show that the universe bounces $(\dot{a}=0)$ at $a=\sqrt{\frac{\left|p_{\phi}\right| \iota 3 \sqrt{3}}{2 \gamma c}}$ and, consequently, that there is no Big Bang singularity, because the range of $a(t)$ is $\left[\sqrt{\frac{\left|p_{\phi}\right| \iota 3 \sqrt{3}}{2 \gamma c}},+\infty\right), \forall t \in \mathbb{R}$. In fact, for earlier times the scalar factor is very big, then it decreases and, when it arrives at the turning value, it finally increases forever.

To properly compare with previous work, a second remark is in order. In [4], the modified Friedmann equation was modified once again, with the aim to obtain a constant critical density. To do this, the authors considered the critical density at the bouncing point $\rho_{\text {bounce }} \equiv \rho_{c}\left(\sqrt{\frac{\left|p_{\phi}\right| \iota 3 \sqrt{3}}{2 \gamma c}}\right)=$ 
$\left(\frac{3 c^{4}}{8 \pi G \iota^{2} \mu^{2}}\right)^{3 / 2} \frac{\sqrt{2}}{\left|p_{\phi}\right|}$, and modified the Friedmann equation as follows:

$$
\frac{\dot{a}^{2}}{a^{2}}=\frac{2 \rho}{\gamma^{2}}\left(1-\frac{\rho(a)}{\rho_{\text {bounce }}}\right) .
$$

Now, we consider the general case of a barotropic fluid with an equation of state $p=\omega \rho$. Then, the density is given by $\rho(a)=\rho_{0}\left(a / a_{0}\right)^{-3(\omega+1)}$. The modified Friedmann equation tells us that the following relation must be satisfied $\rho_{c}(a) \geq \rho(a)$, which shows that, when $\omega>-1 / 3$, the scale

factor belongs in the domain $\left[a_{\text {bounce }}, \infty\right)$, where we have defined $a_{\text {bounce }} \equiv\left(\frac{2 \iota^{2} \mu^{2} \rho_{0} a_{0}^{3(\omega+1)}}{\gamma^{2} c^{2}}\right)^{\frac{1}{3 \omega+1}}$. This means that the Big Bang singularity that appears in classical cosmology when one considers a barotropic fluid with $\omega>-1$ is actually avoided by using loop cosmology, for $\omega>-1 / 3$.

Finally we want to stress the noticeable fact that all results here obtained are at the classical level (followed by proper regularization). We have not used a quantized Hamiltonian to obtain the bounces, but only the quantum nature of the geometry in order to build the regularized Hamiltonian that depends on a parameter, and to fix this one. The quantum Hamiltonian theory will probably reproduce, in an approximate way (with corrections of order $\hbar$ ) all these results, in particular, the right bounces. Of course the situation is not completely clear at this stage, since we have not taken into account the possible role of additional quantum corrections and it is not possible to know if, in the proximity of the bounce, all of them can still be kept small. But what is really important from the considerations above is to understand that, in order to get rid of the singularities, the regularized Hamiltonian of loop gravity suffices, and there is no need to invoke the full quantum version of the theory.

\section{NEW PHASE SPACE VARIABLES IN LOOP QUANTUM COSMOLOGY}

We have shown above that, when one considers a barotropic fluid with equation of state $p=\omega \rho$, the Big Bang singularity is absent from loop cosmology for any $\omega>-1 / 3$. However, it is well-known that, in standard cosmology, the Big Bang appears for $\omega>-1$. In order to avoid that singularity in LQC for all possible values of $\omega$ and to remove a problem that appears in the old quantization of LQC (for details, see $[8,9]$ ), one needs to introduce new canonically conjugate variables, namely $V \equiv a^{3}$ and $\beta \equiv \iota \dot{a} / a$, which satisfy $\{\beta, V\}=\frac{3 \iota}{\gamma^{2}}[8,9]$. And, also, to consider the holonomies $h_{j}(\lambda) \equiv$ $e^{-i \frac{\lambda \beta}{2 c} \sigma_{j}}$, where now $\lambda$ is a parameter with dimensions of length, which is determined invoking the 
quantum nature of the geometry, that is, identifying it with the minimum eigenvalue of the area operator in LQG $\lambda \equiv \sqrt{2 \sqrt{3} \pi \iota} l_{p}$.

Once again, one may use the general formulae of loop gravity to obtain the gravitational regularized Hamiltonian in the flat case [2-4]:

$$
\begin{gathered}
H_{\text {grav }, \text { reg }} \equiv-\frac{\hbar^{2} c}{32 \pi^{2} l_{p}^{4} \iota^{3}} \frac{V}{\lambda^{3}} \sum_{i, j, k} \varepsilon^{i j k} \operatorname{Tr}\left[h_{i}(\lambda) h_{j}(\lambda) h_{i}^{-1}(\lambda)\right. \\
\left.\times h_{j}^{-1}(\lambda) h_{k}(\lambda)\left\{h_{k}^{-1}(\lambda), V\right\}\right]=-\frac{\gamma^{2} c^{2}}{2 \iota^{2} \lambda^{2}} V \sin ^{2} \frac{\lambda \beta}{c} .
\end{gathered}
$$

Then, taking this regularized Hamiltonian as the gravitational part of the full one, the complete Hamiltonian will be given by [8]

$$
H_{r e g} \equiv-\frac{\gamma^{2} c^{2}}{2 \iota^{2} \lambda^{2}} V \sin ^{2} \frac{\lambda \beta}{c}+\rho(a) V
$$

which coincides with the classical one, namely $H \equiv-\frac{\gamma 2}{2} \dot{a}^{2} a+\rho(a) a 3$, when $\lambda \rightarrow 0$.

The dynamical equation for the scalar factor reads

$$
\dot{a}=\left\{a, H_{\text {reg }}\right\}=\frac{c a}{2 \lambda \iota} \sin \frac{2 \lambda \beta}{c} .
$$

Imposing the Hamiltonian constraint, $H_{r e g}=0$, we obtain the following modified Friedmann equation

$$
\frac{\dot{a}^{2}}{a^{2}}=\frac{2 \rho}{\gamma^{2}}\left(1-\frac{\rho(a)}{\rho_{c}}\right)
$$

where now the critical density is a constant, with value

$$
\rho_{c} \equiv \frac{\gamma^{2} c^{2}}{2 \lambda^{2} \iota^{2}}=\frac{\sqrt{3} \hbar c}{16 \pi^{2} \iota^{3} l_{p}^{4}}=\frac{\sqrt{3} c^{7}}{16 \pi^{2} \iota^{3} G^{2} \hbar} .
$$

Note that the modified Friedmann equation (10) is actually invariant under the scaling $a \rightarrow C a$, what is a very essential property of flat FRW space-time. The same does not happen, however, with Eq. (4), this being one of the main shortcomings of the old quantization.

From (10) it follows that, for $\omega>-1$, the scalar factor belongs in the domain $\left[a_{\text {bounce }}, \infty\right)$, with $a_{\text {bounce }} \equiv\left(\frac{2 \iota^{2} \lambda^{2} \rho_{0} a_{0}^{3(\omega+1)}}{\gamma^{2} c^{2}}\right)^{\frac{1}{3(\omega+1)}}$, what means that the Big Bang singularity which appears in classical cosmology for a barotropic fluid with $\omega>-1$ will be absent, for all values of $\omega$, in the framework of regularized loop cosmology, provided the new variables are used. Moreover, also with the use of 
these new variables, it was seen in [10-12] that, when one considers a barotropic fluid with $\omega<-1$, the Big Rip singularity is avoided too. In order to prove this, note that from (10) we have $\rho_{c}>\rho(a)$, and then $a<\left(\frac{\rho_{c}}{\rho_{0}} a_{0}^{3|\omega+1|}\right)^{\frac{1}{3|\omega+1|}}$, what completes our statement.

To summarize, we have rigorously proven in loop gravity, for the cases explicitly considered, that using the classical regularized Hamiltonian one is able to avoid the singularities. No quantization of the Hamiltonian was actually used above, only the quantum nature of the geometry was invoked (the loop, or area operator, cut off) in order to construct the regularized Hamiltonian and to fix the parameter on which it depends. This is quite different from the quantum effective formulation for FRW cosmologies as discussed in [17], or from Schutz's formalism as applied to quantum cosmological fluids [18-20], where one does quantize the Hamiltonian in order to be free from singularities. It is important to realize, once more, that it is the regularization of the classical gravitational Hamiltonian introduced in loop gravity what avoids the singularities, rather than the quantum effects due to its quantization. This has been overlooked in several papers, where it was claimed that quantum effects, provided by the quantum Hamiltonian, were in fact essential in order to avoid the singularities $[4,8,10,12]$. Note that in these approximations the authors start, from the very beginning, from the quantum theory and then, using semi-classical techniques, coherent states techniques, etc. an effective Hamiltonian is obtained [9, 13-15] which, in fact, coincides in essence with our Hamiltonian (8). This is maybe the reason why it was plainly concluded in those papers that quantum effects provided by the quantum Hamiltonian in LQC were the ones responsible for avoiding the singularities, what we have here proven not to be the case.

\section{CONCLUSION}

We have shown in this paper that it is the regularization of the classical Hamiltonian performed in loop gravity what is already able to avoid the important singularities of the initial theory, rather than quantum effects arising after the quantization of the Hamiltonian. In this sense, one can say that what actually resolves the Big Bang and the Big Rip singularities is just the regularized loop cosmology, rather than the quantum version (LQC). Such finding, far from being a draw back, constitutes a key manifestation of the intrinsic potential of loop gravity, as compared with competing theories. In other words, the capability to avoid the singularities actually lies in the loop-cosmology (geometrical) paradigm itself, what makes this theory even more powerful than it had been thought to be. 
Acknowledgements. This investigation has been supported in part by MICINN (Spain), projects MTM2008-06349-C03-01, FIS2006-02842 and grant PR2009-0314, and by AGAUR (Generalitat de Catalunya), contract 2005SGR-00790. EE's research was performed while on leave at Department of Physics and Astronomy, Dartmouth College, 6127 Wilder Laboratory, Hanover, NH 03755, USA.

[1] T. Thiemann, Class. Quantum Grav. 15, 1281 (1998).

[2] A. Ashtekar, M. Bojowald and J. Lewandowski, Adv. Theor. Math. 7, 233 (2003).

[3] T. Thiemann, Introduction to modern canonical quantum general relativity, gr-qc/0110034 (2001).

[4] A. Ashtekar, T. Pawlowski and P. Singh, Phys. Rev. D73, 124038 (2006).

[5] A. Corichi and P. Singh, Phys. Rev. D78, 024034 (2008).

[6] P. Singh, Transcending Big Bang in Loop Quantum Cosmology: Recent Advances, gr-qc/0901.1301 (2009).

[7] P. Singh, Phys. Rev. D73, 063508 (2006).

[8] P. Singh, Are loop quantum cosmos never singular?, gr-qc/0901.2750 (2009).

[9] V. Taveras, Phys. Rev. D78, 064072 (2008).

[10] M. Sami, P. Singh and S. Tsujikawa, Phys. Rev. D74, 043514 (2006).

[11] T. Naskar and J. Ward, Phys. Rev. D76, 063514 (2007).

[12] D. Smart and B. Gumjudpai, Phys. Rev. D76, 043514 (2007).

[13] K. Vandersloot, Phys. Rev. D71, 103506 (2005).

[14] G. Date and G.M. Hossain, Class. Quantum Grav. 21, 4941 (2004).

[15] K. Banerjee and G. Date, Class. Quantum Grav. 22, 2017 (2005).

[16] P. Singh and K. Vandersloot, Phys. Rev. D72, 084004 (2005).

[17] J. Haro and E. Elizalde, J. Phys. A: Math. Theor. 42, 202002 (2009).

[18] F.G. Alvengara, J.C. Fabris, N.A. Lemos and G.A. Monerat, Gen. Rel. Grav. 34, 651 (2002).

[19] A.B. Batista, J.C. Fabris, S.V.B. Gonçalves and J. Tossa, Phys. Lett. A283, 62 (2001).

[20] A.B. Batista, J.C. Fabris, S.V.B. Gonçalves and J. Tossa, Phys. Rev D65, 063519 (2002). 